Supporting Information

\title{
Three-Dimensional-Printed Silica Aerogels for Thermal Insulation by Directly Writing Temperature-Induced Solidifiable Inks
}

Lukai Wang, Junzong Feng*, Yi Luo, Zhenhao Zhou, Yonggang Jiang, Xuanfeng Luo, Lin Xu, Liangjun Li, Jian Feng*

Science and Technology on Advanced Ceramic Fibers and Composites Laboratory, College of Aerospace Science and Engineering, National University of Defense Technology, 109 De Ya Rd, Changsha, Hunan, 410073, P.R. China

*Corresponding Authors:

Jian Feng, E-mail: fengj@nudt.edu.cn;

Junzong Feng, E-mail: junzongfeng@nudt.edu.cn

This PDF file includes:

Captions for Videos S1 to S4

Figures S1 to S10

Table S1

Other Supplementary Materials for this manuscript include the following:

Videos S1 to S4 


\section{Video list:}

Video S1. 3D printing of concave-shaped silica aerogel heat insulation shield. (MP4)

Video S2. 3D printing of silica microgrids. (MP4)

Video S3. 3D printing of triangular silica aerogel monolith. (MP4)

Video S4. 3D printing of silica aerogels with trapezoidal pyramid shapes. (MP4)

\section{Figures:}
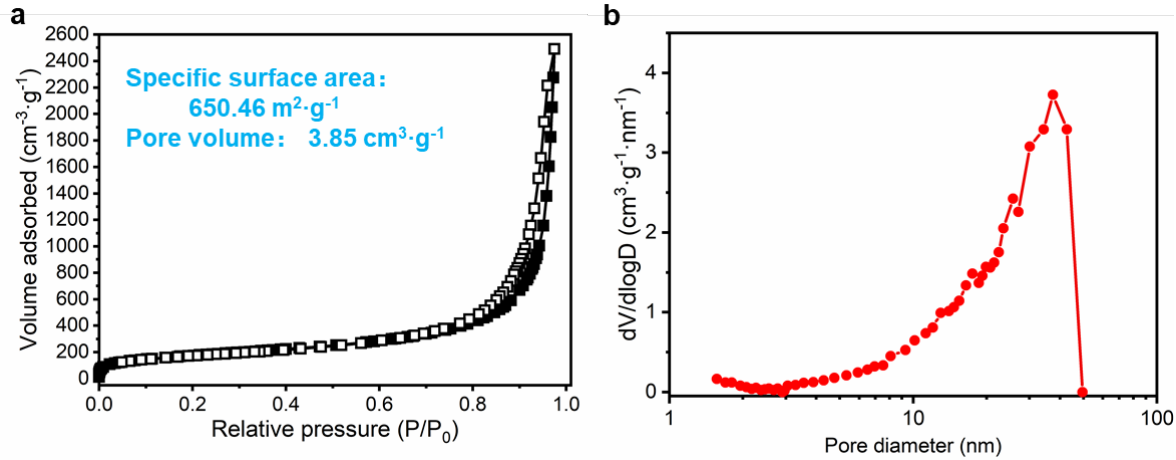

Figure S1. Pore structure features of ball-milling silica aerogel powders. (a) $\mathrm{N}_{2}$ adsorption-desorption isotherms. (b) Pore size distribution.

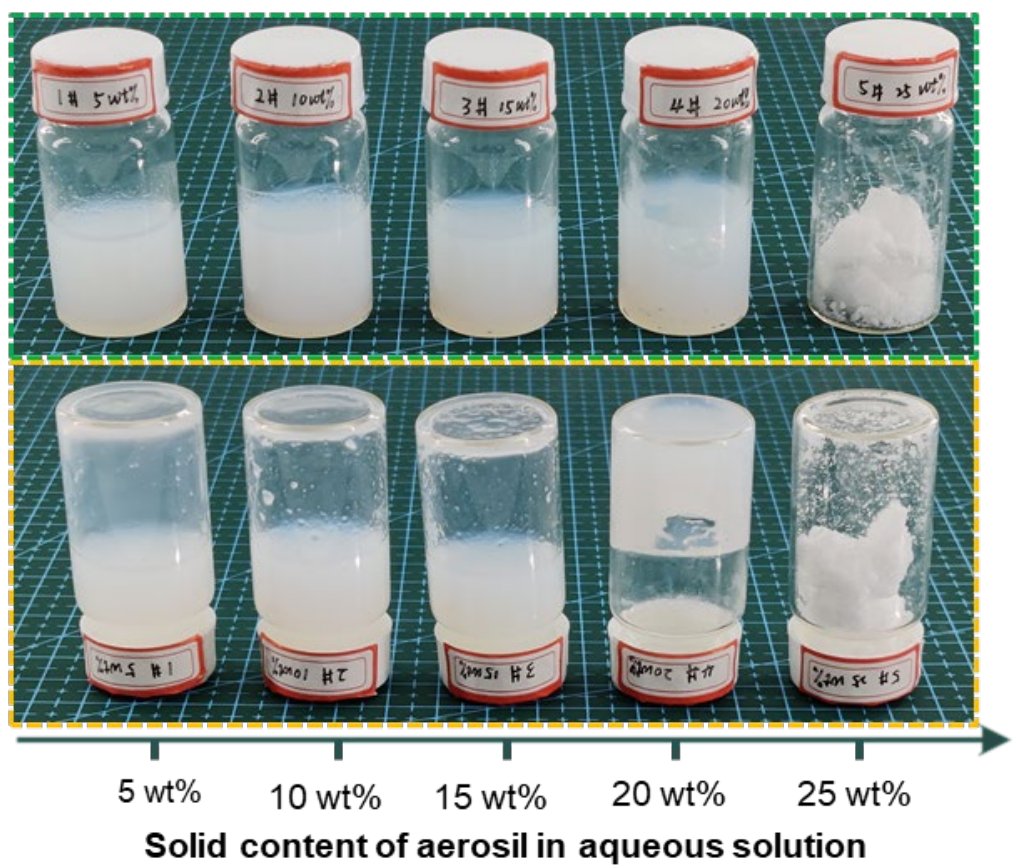

Figure S2. Aerosil aqueous solution with different solid contents. 

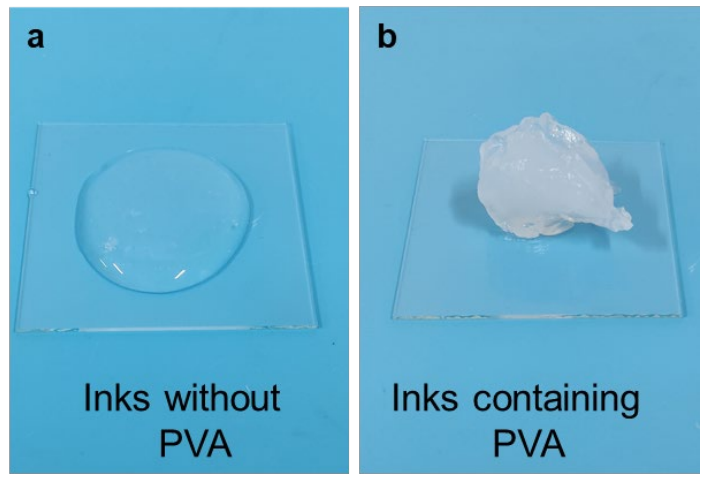

Figure S3. Comparison photographs of rheological behaviors of different silica inks. (a) Ink without $0.04 \mathrm{wt} \%$ PVA polymers. (b) Ink containing $0.04 \mathrm{wt} \%$ PVA polymers.

a

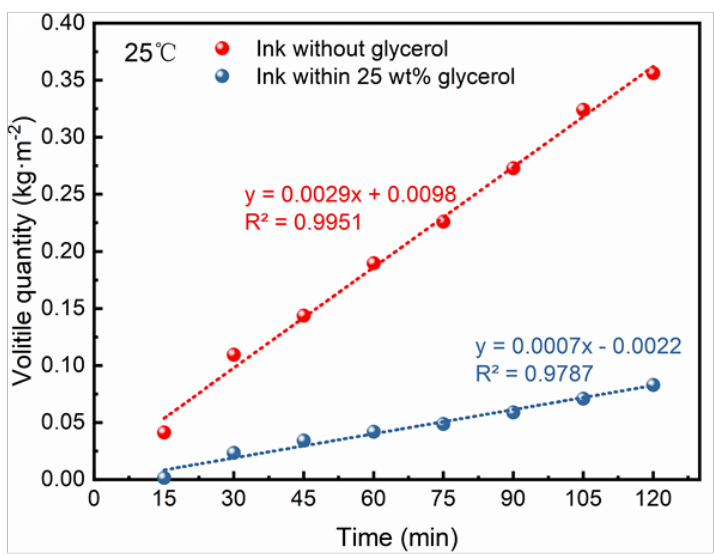

C

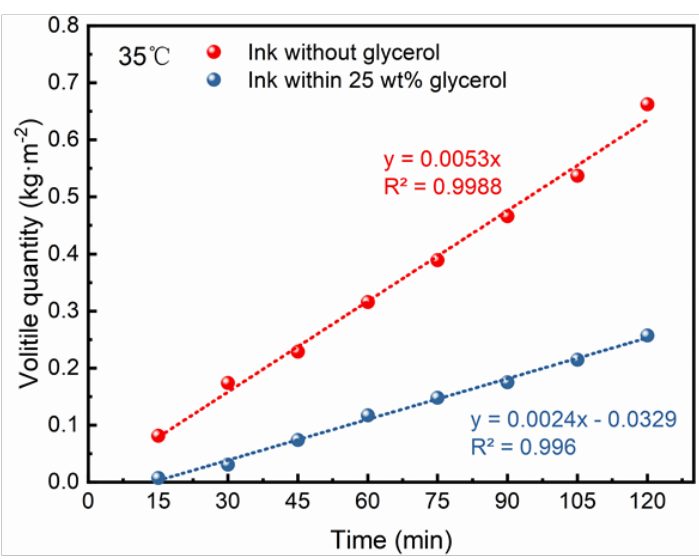

b

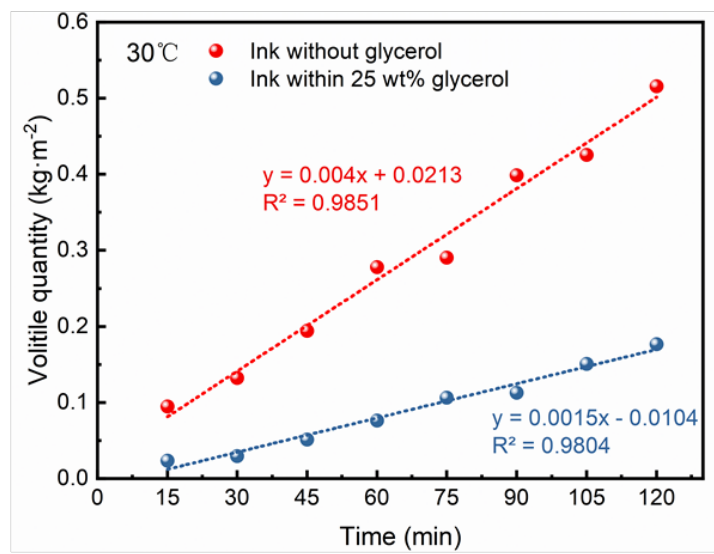

d
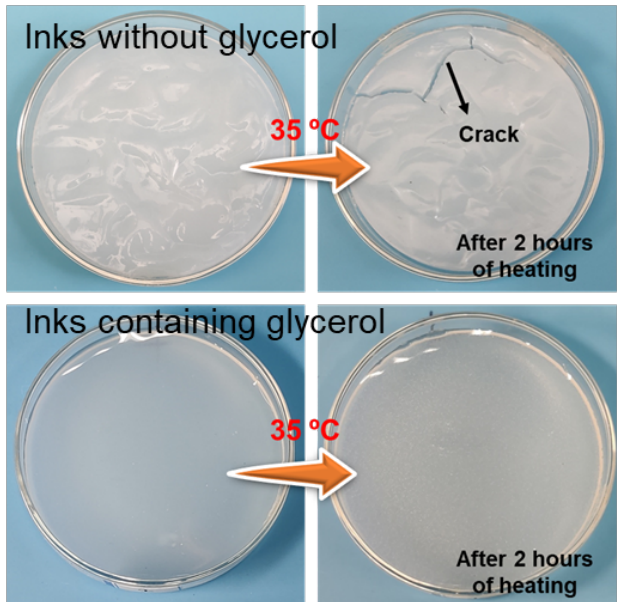

Figure S4. Volatilization rates of silica inks with or without glycerol solvent heating at (a) $25^{\circ} \mathrm{C}$, (b) $30{ }^{\circ} \mathrm{C}$, and (c) $35^{\circ} \mathrm{C}$ for 2 hours in an atmospheric environment. (d) Comparison photographs of different silica inks before and after heating. 


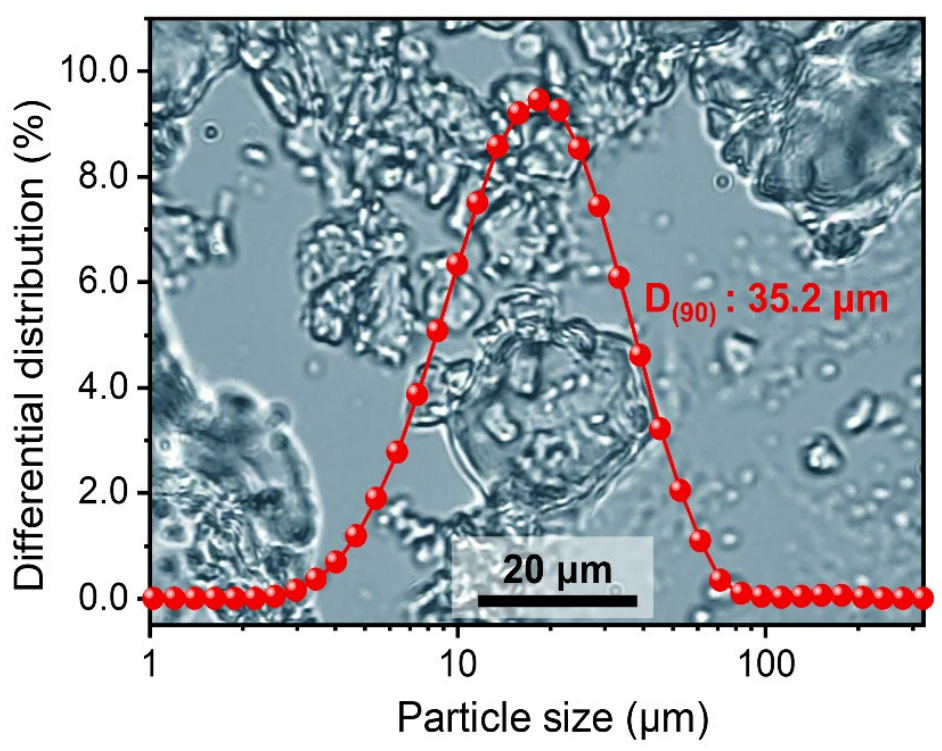

Figure S5. Particle size distribution of silica aerogel powders.
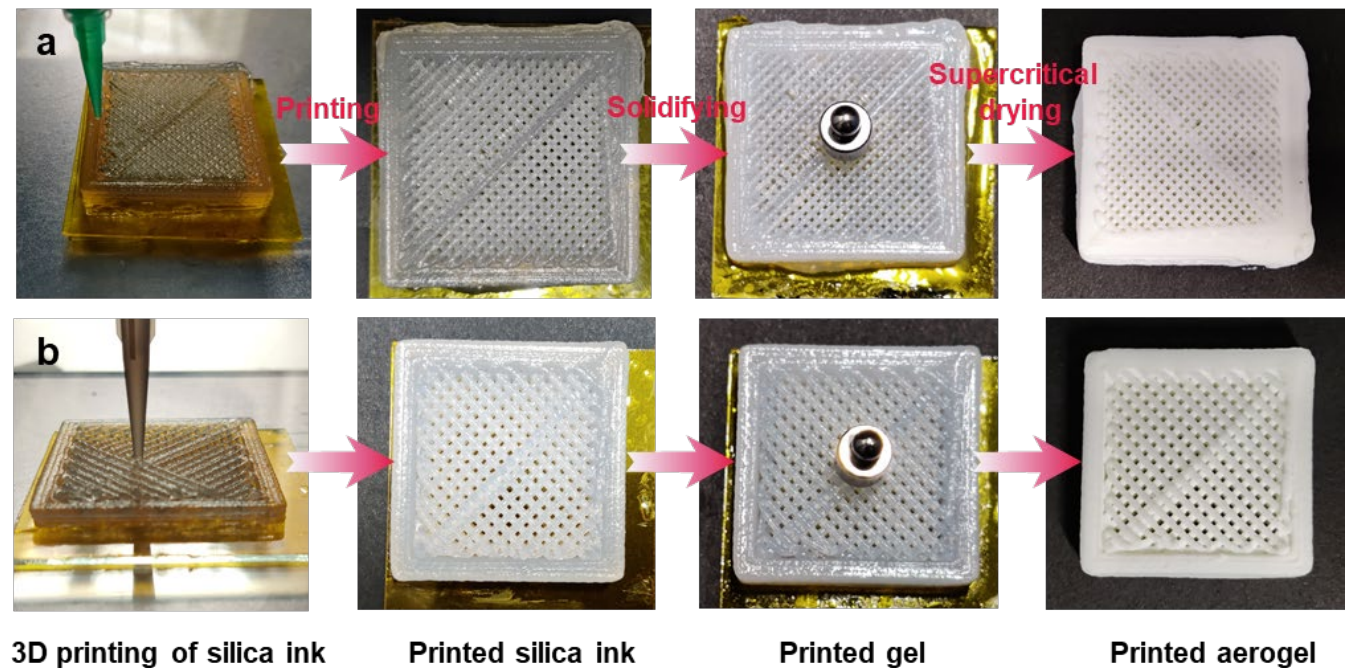

Figure S6. Thermal-solidifying 3D printing processes of silica aerogel microgrids using (a) a $0.84 \mathrm{~mm}$ nozzle diameter and (b) a $1.2 \mathrm{~mm}$ nozzle diameter. 

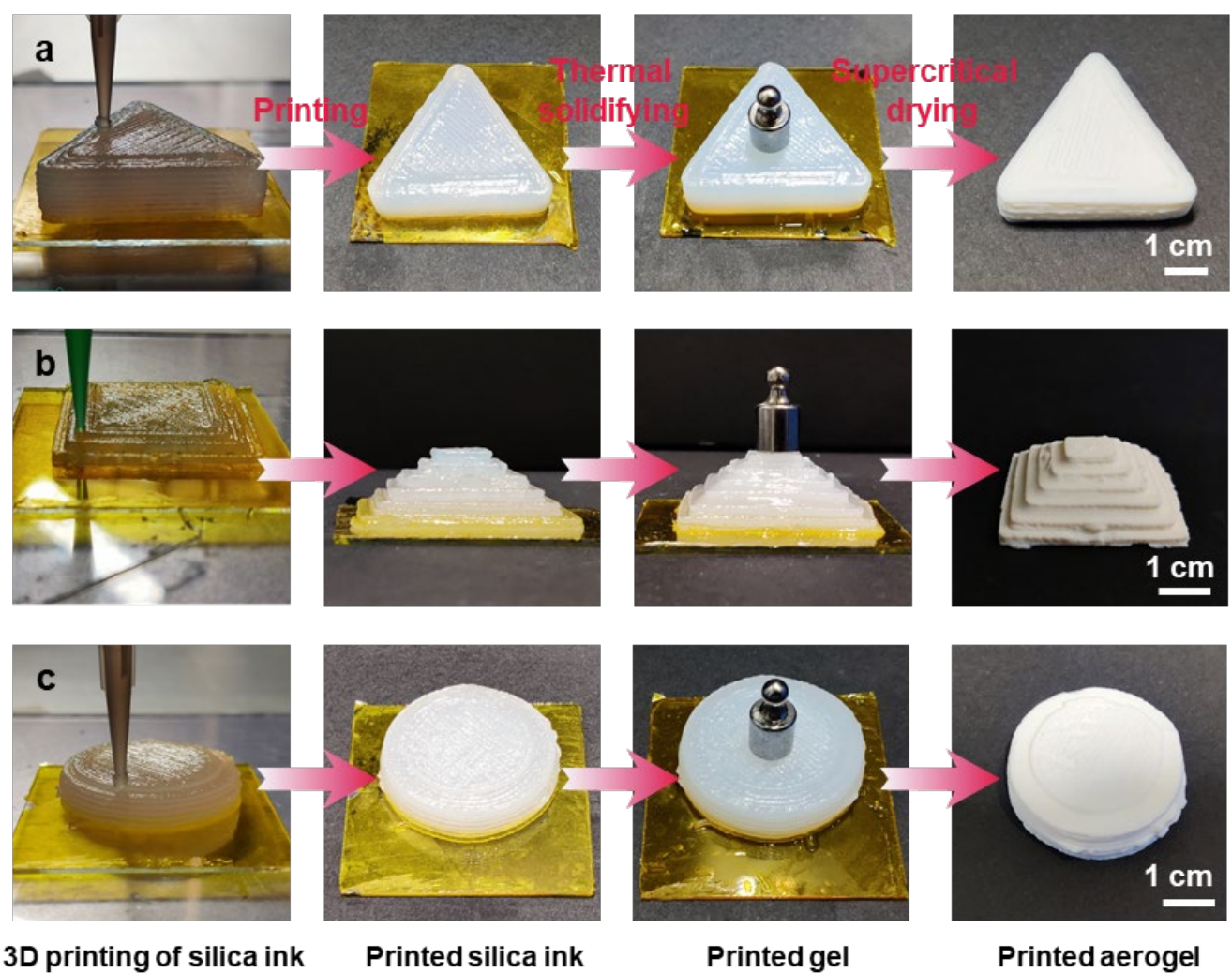

Figure S7. Thermal-solidifying 3D printing processes of silica aerogels with different architectures and patterns. (a) Triangular monolith. (b) Trapezoidal pyramid. (c) Cylindrical monolith.

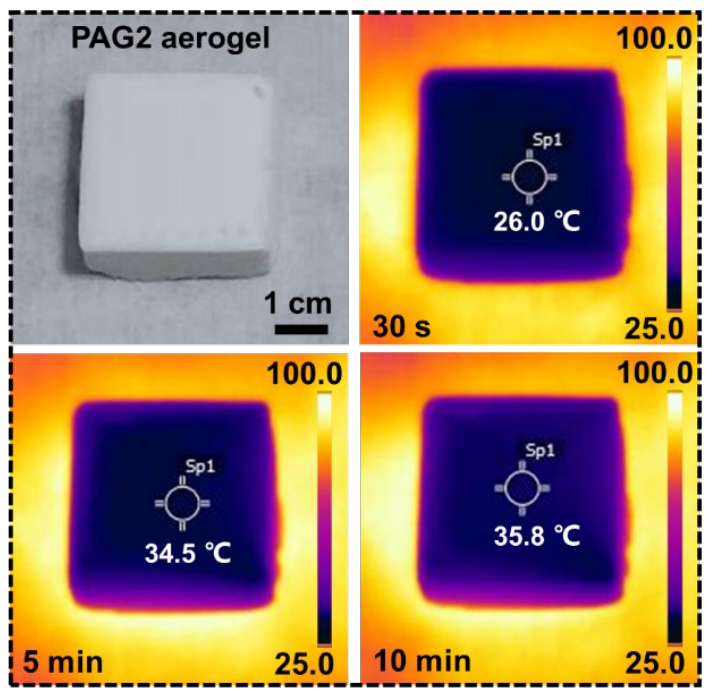

Figure S8. Optical and infrared images of PAG2 aerogels (thickness: $12 \mathrm{~mm}$ ) on a $100{ }^{\circ} \mathrm{C}$ heating stage for different times. 


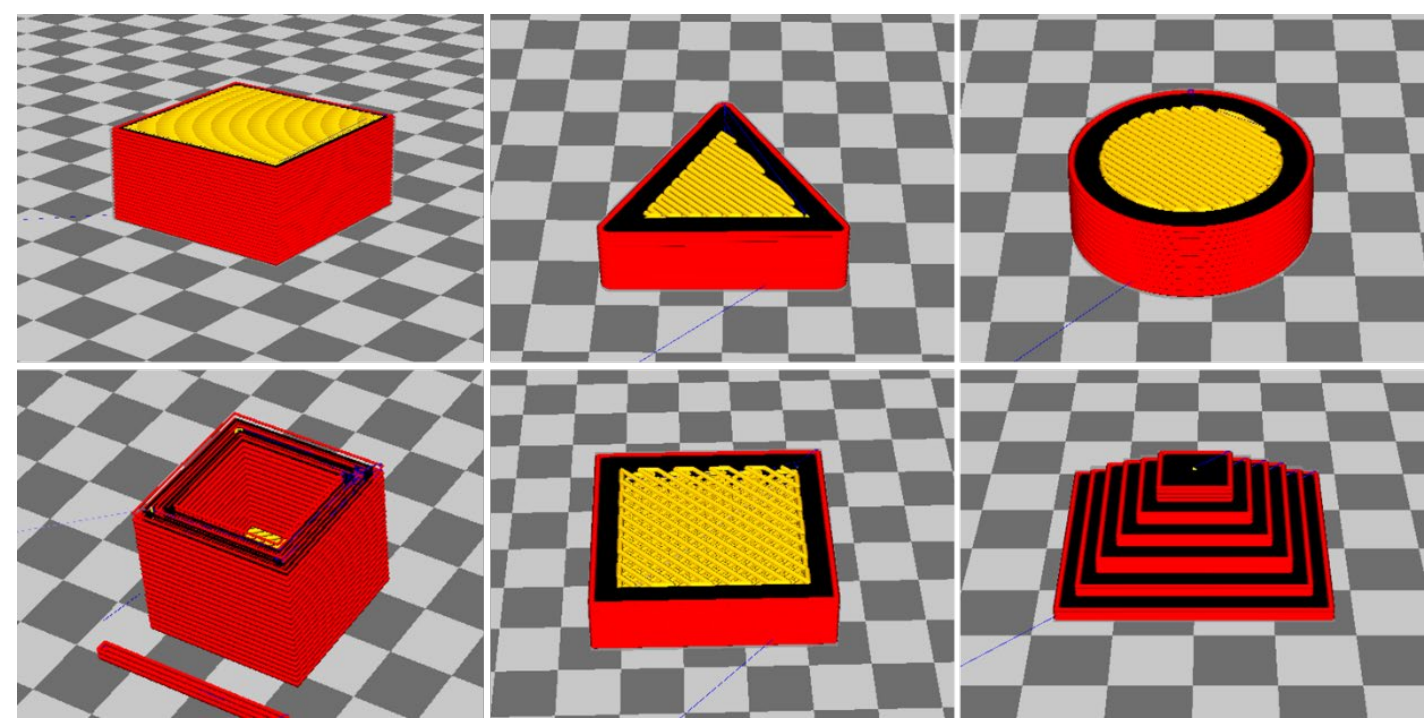

Figure S9. 3D models sliced by the Smart Slicer software.

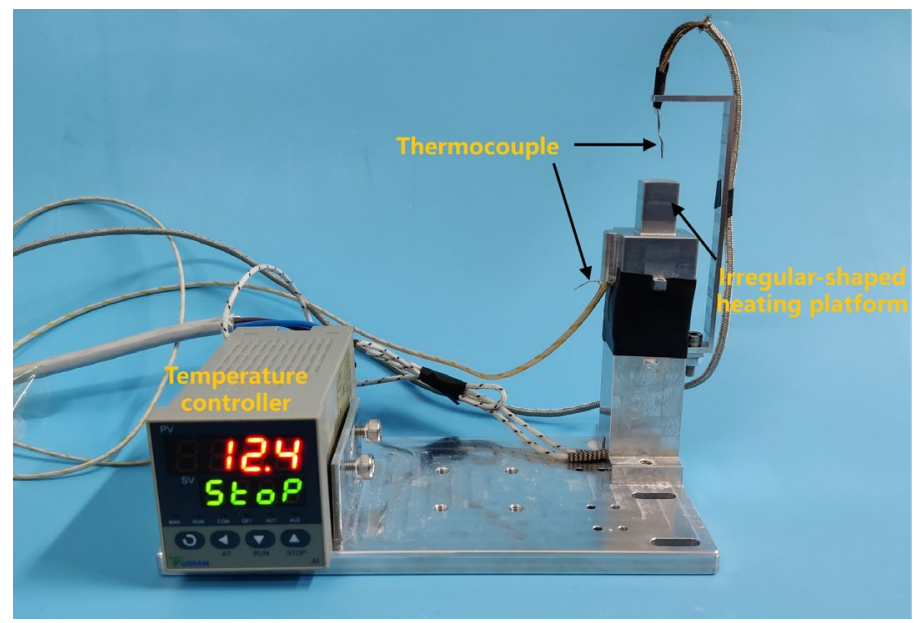

Figure S10. Customized heating platform for measuring the cold-side and hot-side temperatures of silica aerogel heat shield.

\section{Table:}

Table S1. Atomic percentage of different aerogel samples obtained by analyzing XPS data.

\begin{tabular}{cccc}
\hline Atom & PAS2 aerogel & $\begin{array}{r}\text { PAG2 aerogel } \\
\text { Atomic Percentage }\end{array}$ & $\begin{array}{c}\text { PAS2H aerogel } \\
(\%)\end{array}$ \\
\hline Si2p & 31.3 & 31.4 & 31.5 \\
C1s & 3.6 & 2.9 & 7.8 \\
O1s & 65.1 & 65.7 & 60.7 \\
\hline
\end{tabular}

